\title{
Effectiveness of hydromorphone hydrochloride against cancer patient dyspnea
}

\author{
Daisuke Tanada, Hiroki Miyawaki, Takako Nagai, Kazuma Hashimoto, \\ Norihiko Sukenaga, Yumiko Takao, Munetaka Hirose \\ Department of Anesthesiology and pain medicine, Hyogo college of Medicine, Hyogo, Japan
}

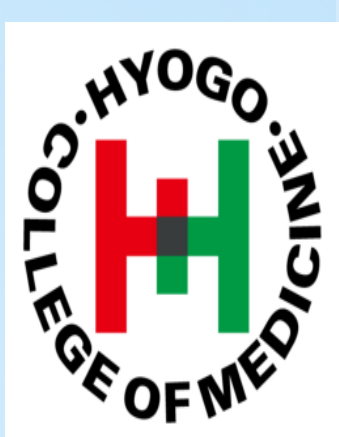

\section{【Introduction】}

In Japan, June 2017, sustained release hydromorphone hydrochloride (hereinafter abbreviated as $\mathrm{HyH}$ ) and the rapid release $\mathrm{HyH}$ were released for the cancer pain. HyH intravenous injection was released in July 2018. Our hospital also used cases for cancer pain accompanied by dyspnea symptoms. There are few reports on the effectiveness of $\mathrm{HyH}$ on respiratory distress symptoms in Japan. We have reported on the effectiveness of $\mathrm{HyH}$ against respiratory symptoms associated with cancer in Japanese patients.

\section{【Methods】}

We investigate the effectiveness of $\mathrm{HyH}$ on dyspnea symptoms caused by cancer. The subjects retrospectively examined cases using $\mathrm{HyH}$ for cases with cancer pain accompanied by dyspnea among palliative care team intervention cases from July 2017 to September 2018. The administration of $\mathrm{HyH}$ alleviated respiratory symptoms, and the case that the patient wanted to continue the administration was regarded as the effective case.

\section{【Results】}

In July 2017 - September 2018, the number of palliative care team intervention cases was 250 cases, of which 32 cases were using $\mathrm{HyH}$ for cases with cancer pain accompanied by dyspnea. The HyH preparations used were duplicated, 22 sustained release preparations, 21 rapid release preparations and 6 intravenous preparation.

Cancer types were lung cancer: 10 cases, malignant pleural mesothelioma: 11 cases, metastatic lung tumor / pleural dissemination: 11 cases. The effective rate for dyspnea was $69 \%$ (22/32).

\section{【Conclusions】}

In Japan, Systemic administration of morphine to dyspnea of cancer patients is recommended by JSPM: Japanese Society for Palliative Medicine. But HyH recommendations have not been mentioned by JSPM yet. ESMO: European Society for Medical Oncology Clinical practice Guidelines recommend morphine and $\mathrm{HyH}$ for treatment of dyspnea of advanced cancer patient.

In our study, $\mathrm{HyH}$ showed a high efficacy rate of $69 \%$ against dyspnea. HyH is considered to be effective in respiratory symptoms associated with cancer as well as morphine in Japanese patients.

Advantages of $\mathrm{HyH}$ sustained release formulation reduces the oral number borne by once daily formulation for opioid naïve patient can start from a low dose $(\mathrm{HyH}$ sustained release formulation $2 \mathrm{mg}$ : oral morphine converted $10 \mathrm{mg} /$ day), fast release formulation is fast acting not inferior to immediate release Oxycodone, drug interactions due to glucuronidation main metabolic pathway is small, the activity of the metabolite is a very low value of for such can be used in renal dysfunction patients compared to morphine hydrochloride Can be mentioned.

Until now, there were many cases that hesitated to use morphine hydrochloride for the purpose of alleviating symptoms of dyspnea in cases with impaired renal function. However, the $\mathrm{HyH}$ can be used for cases with decreased renal function compared to morphine hydrochloride, it is possible to use it relatively carelessly for patients with dyspnea symptoms.

$\mathrm{HyH}$ seems to be increasingly used for respiratory symptoms associated with cancer in Japan in the future.

\section{Structure and formation of major opioids}

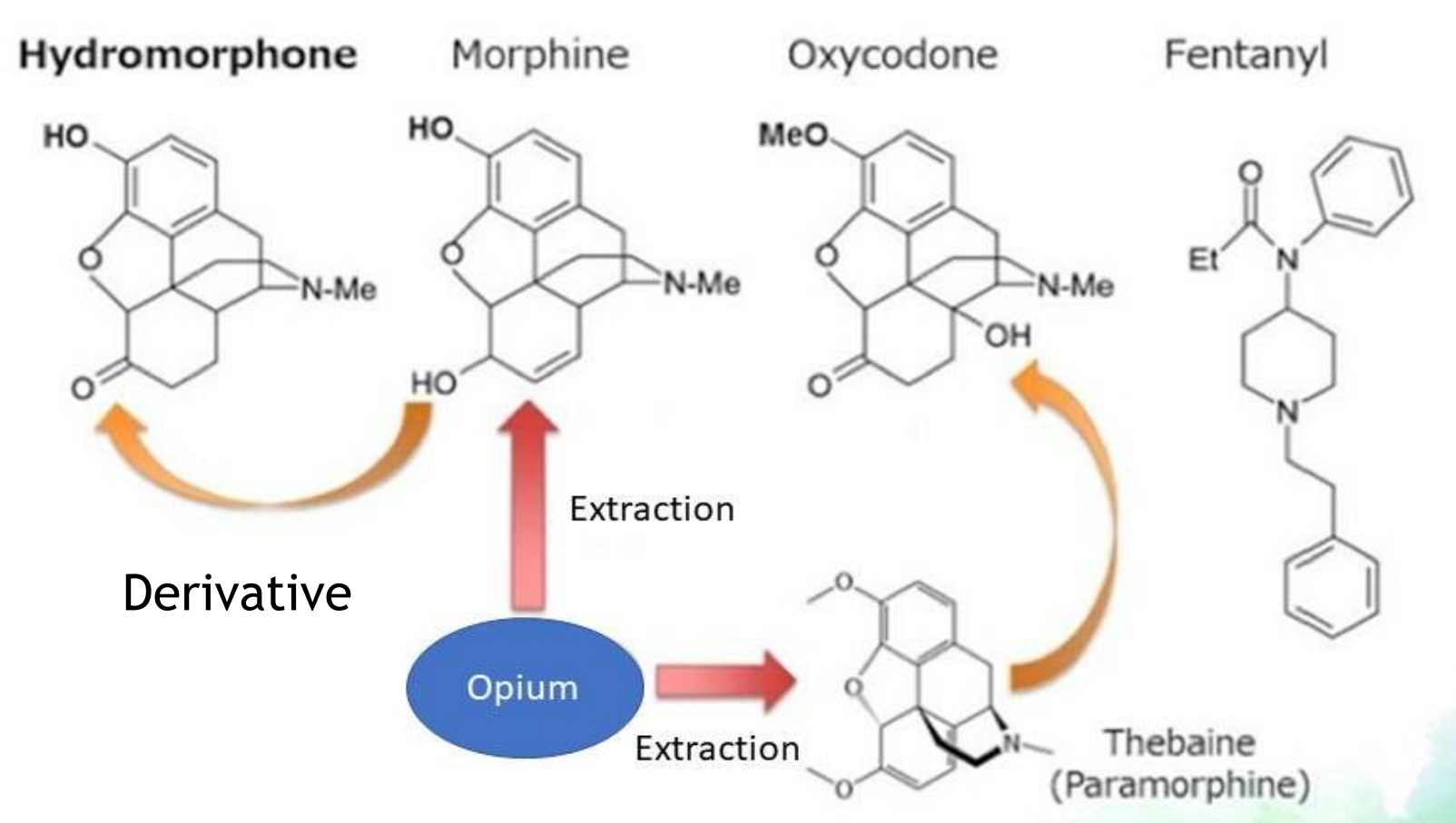

\begin{tabular}{|l|l|}
\hline Cancer type & Cases \\
\hline $\begin{array}{l}\text { lung cancer } \\
\text { Malignant pleural mesothelioma }\end{array}$ & 10 cases \\
\hline $\begin{array}{l}\text { Metastatic lung tumor, } \\
\text { pleural dissemination } \\
\text { total }\end{array}$ & 11 cases \\
\hline
\end{tabular}

Characteristics of $\mathrm{HyH}$

- Sustained-release $\mathrm{HyH}$ is a once-daily preparation

- The immediate release $\mathrm{HyH}$ is as immediate as immediate release Oxycodone

- HyH metabolic pathway is mainly glucuronidation so it can also be used for renal dysfunction cases

- HyH do not inhibit CYP1A2, 2A6, 2B6, 2C8, 2C9, 2C19, 2D6, 2E1, $3 \mathrm{~A} 4$ / 5 and do not induce CYP1A2, 2B6, 3A4, so it has low drug interaction and easy to use

Guidelines for alleviating respiratory symptoms in cancer patients (JSPM: Japanese Society for Palliative Medicine 2016

Q: Is morphine effective for cancer patients who complain of dyspnea?

A: Systemic administration of morphine is recommended for dyspnea in cancer patients.

1B (strong recommendation, moderate evidence)

Treatment of dyspnea of advanced cancer patient:

ESMO: European Society for Medical Oncology Clinical practice Guidelines

Stating doses of opioids for the palliation of dyspnea

Opioid

Morphine

Hydromorphone
Stating dose of opioid negative

2.5-5mg/4hr p.o.

$1-2.5 \mathrm{mg} / 4 \mathrm{hr}$ s.c.

$1.3 \mathrm{mg} / 4 \mathrm{hr}$ p.o. $0.2-0.5 \mathrm{mg} / 4 \mathrm{hr}$ s.c.
Starting dose of concomitant opioid intake

Regular opioid dose + $1 / 6$ of the daily opioid intake

Regular opioid dose + $1 / 6$ of the daily opioid intake 